\title{
Alterações Audiológicas em Pacientes com Doença Falciforme Acompanhados em um Hospital Escola.
}

\section{Ana Elisa de Faveri*, Thais A. Diniz Hein, Rafael V. Lucena, Jorge R. Paschoal, Maria Isabel Ramos do Amaral.}

\section{Resumo}

Diversos estudos sugerem que pacientes com doença falciforme tem um risco aumentado para desenvolver Deficiência Auditiva Neurossensorial (DANS) devido a hipóxia no órgão de Corti causada por hemácias falciformes. O objetivo deste trabalho foi o de caracterizar a audição de um grupo de 25 sujeitos diagnosticados com Doença Falciforme a partir de métodos convencionais e complementares da avaliação auditiva. Concluiu-se que houve uma alta prevalência de PANS na amostra. Além disso, houve maior prevalência de alterações na Audiometria de Altas Frequências e nas Emissões Otoacústicas do que na avaliação audiológica convencional, sugerindo que são métodos adequados para a detecção precoce de perdas auditivas no grupo estudado.

\section{Palavras-chave: \\ Doença Falciforme, Perda Auditiva Neurossensorial, Avaliação Auditiva.}

\section{Introdução}

A Doença Falciforme (DF) é definida como uma doença em que ocorre a transformação da hemoglobina normal $(\mathrm{HbA})$ para a hemoglobina falcizada $(\mathrm{HbS})$, a qual acarreta em significativo impacto na vida do indivíduo. Dentre as possíveis alterações, destaca-se a perda auditiva neurossensorial (PANS) devido a hipóxia no órgão de Corti. (1)

A literatura tem destacado métodos complementares para avaliação auditiva como a Audiometria de Altas Frequências (AAF) e as Emissões Otoacústicas (EOA) em relação a avaliação convencional, pois permitem a avaliação e detecção precoce de alterações auditivas. ${ }^{(2)} 0$ objetivo foi o de caracterizar e comparar a audição de sujeitos com diagnóstico de DF por meio de métodos avaliativos convencionais e complementares.

\section{Resultados e Discussão}

Tipo e local do estudo: descritivo, quantitativo de corte transversal (CEP: nº 2.338.277) / Laboratório de Audiologia do DDHR - FCM/Unicamp.

Parceria: Departamento de ORL e DDHR - Unicamp. Local de seleção: Ambulatório de Hemoglobinopatias - Unicamp. Critérios de inclusão: idade entre 18 e 60 anos, ambos os sexos, diagnóstico de DF confirmado.

Procedimentos: Anamnese audiológica; Avaliação audiológica básica; Audiometria de Altas Frequências (AAF) e Emissões Otoacústicas Transientes (EOAT) e Produto Distorção (EOAPD).

Amostra: 25 sujeitos, sendo 15 homens (60\%) e 10 mulheres (40\%), média de idade de 42,9 anos e desvio padrão 9,8 .

A alteração da hemoglobina na forma homozigótica (HbSS) foi a mais prevalente (19/76\%) e o sintoma auditivo mais prevalente foi a tontura (15/60\%).

Do total da amostra, 19 sujeitos (76\%) apresentaram audição caracterizada como dentro dos padrões de normalidade.
Tabela 1. Média dos limiares tonais da AAF por orelha.

\begin{tabular}{|l|l|l|l|l|l|}
\hline & $\mathbf{9 K H z}$ & $\mathbf{1 K H z}$ & $\mathbf{1 1 , 2 K H z}$ & $\mathbf{1 2 , 5 K H z}$ & $\mathbf{1 4 K h z}$ \\
\hline OD & 33,8 & 36,4 & 43,4 & 52,2 & 57,4 \\
\hline OE & 29,8 & 34 & 41,4 & 44,8 & 52,8 \\
\hline
\end{tabular}

Tabela 2. Média da relação sinal/ruído referente a EOAT, considerando orelha direita e esquerda.

\begin{tabular}{|l|l|l|l|l|l|}
\hline & $\mathbf{1 K H z}$ & $\mathbf{2 K H z}$ & $\mathbf{3 K h z}$ & $\mathbf{4 K h z}$ & $\mathbf{5 K h z}$ \\
\hline OD & 18,58 & 17,98 & 11,79 & 7,84 & 1,32 \\
\hline OE & 19,13 & 16,41 & 9,97 & 8,89 & 2,84 \\
\hline
\end{tabular}

Tabela 3. Média das EOAPD por orelha nas frequências de $500 \mathrm{~Hz}$ a $4 \mathrm{KHz}$.

\begin{tabular}{|l|l|l|l|l|l|l|}
\hline & $\mathbf{5 0 0}$ & $\mathbf{1 K h z}$ & $\mathbf{1 , 5 K H z}$ & $\mathbf{2 K h z}$ & $\mathbf{3 K h z}$ & $\mathbf{4 K h z}$ \\
\hline OD & 6,29 & 16,4 & 17,54 & 18,73 & 16,11 & 14,61 \\
\hline OE & 4,83 & 16,73 & 18,94 & 18,4 & 14,2 & 15,7 \\
\hline
\end{tabular}

Tabela 4. Média das EOAPD por orelha nas frequências de $5 \mathrm{KHz}$ a $10 \mathrm{KHz}$.

\begin{tabular}{|l|l|l|l|l|l|l|}
\hline & $\mathbf{5 K h z}$ & $\mathbf{6 K h z}$ & $\mathbf{7 K h z}$ & $\mathbf{8 K H z}$ & $\mathbf{9 K h z}$ & $\mathbf{1 0 K h z}$ \\
\hline OD & 10,62 & 13,39 & 13,9 & 11,92 & 14,4 & 15,97 \\
\hline OE & 13,28 & 15,7 & 15,69 & 15,13 & 15,02 & 17,44 \\
\hline
\end{tabular}

\section{Conclusões}

Concluiu-se que houve uma alta prevalência de PANS na amostra. Além disso, houve maior prevalência de alterações na Audiometria de Altas Frequências e nas Emissões Otoacústicas do que na avaliação audiológica convencional, sugerindo que são métodos adequados para a detecção precoce de perdas auditivas no grupo estudado.

\section{Agradecimentos}

Ao SAE, pelo financiamento da pesquisa.

[1] COST A, et al. Anemia Falciforme. In: ZAGO, M. A., et. al. Tratado de Hematologia. São Paulo. Editora: Atheneu. p. 205-223. 2013.

[2] WEIGERT, L. L. Estudo da audição em frequências ultra-altas e emissões otoacústicas em pacientes com hemoglobinopatias: diferentes faixas etárias de duração da doença e de exposição ao tratamento. Universidade Federal do Rio Grande do Sul. Tese. Porto Alegre. 2015. 